\title{
Master-equation approach to the study of phase-change processes in data storage media
}

\author{
K. B. Blyuss, P. Ashwin, A. P. Bassom, ${ }^{*}$ and C. D. Wright \\ School of Engineering, Computer Science and Mathematics, University of Exeter, Exeter EX4 4QE, United Kingdom
}

(Received 14 January 2005; published 19 July 2005)

\begin{abstract}
We study the dynamics of crystallization in phase-change materials using a master-equation approach in which the state of the crystallizing material is described by a cluster size distribution function. A model is developed using the thermodynamics of the processes involved and representing the clusters of size two and greater as a continuum but clusters of size one (monomers) as a separate equation. We present some partial analytical results for the isothermal case and for large cluster sizes, but principally we use numerical simulations to investigate the model. We obtain results that are in good agreement with experimental data and the model appears to be useful for the fast simulation of reading and writing processes in phase-change optical and electrical memories.
\end{abstract}

DOI: $10.1103 /$ PhysRevE.72.011607

PACS number(s): 81.10.-h, 81.40.Ef

\section{INTRODUCTION}

Recently there has been a growing interest in developing adequate models of crystallization dynamics of phase-change (PC) materials because of their increasing technological importance in read-write optical and electrical data storage. Both types of storage can utilize PC materials with reversible transformations between amorphous and crystalline states $[1,2]$. Data recording is achieved through writing amorphous dots on a crystalline base by local melting with a focused laser beam or electrical heating. After the heating is withdrawn the melt is quenched in an amorphous state due to high heat conductivity of the substrates surrounding the PC material. Conversely, to erase a dot, a material is heated above the glass transition temperature but below the melting point, and then recrystallization occurs. Some significant differences in optical properties (for optical storage) or electrical properties (for electrical storage) of the crystalline and amorphous states allow the stored data to be read easily. Experiments suggest that crystallization is generally a much slower process than amorphization, and there is also a difference between crystallization of melt-quenched or asdeposited amorphous states [3]. Therefore crystallization is a speed-limiting process for the erasing and rewriting of optical disks, and a good understanding and modeling of crystallization dynamics is vital for the successful development of advanced PC optical and electrical memory devices.

Crystallization of PC materials involves two processes: nucleation and growth. At the initial stage of evolution nucleation leads to the formation of so-called clusters, or embryos, which grow by incorporation of neighboring monomers. A critical cluster size can be identified-its value is a function of a number of variables including the temperature and the competition between surface tension and the free-energy difference between amorphous and crystalline states. The significance of the critical size is that larger clusters will tend to continue to grow while smaller ones will, on

\footnotetext{
*Present address: School of Mathematics and Statistics, University of Western Australia, Crawley 6009, Australia.
}

average, lose their monomer groups. This means that in time these small clusters will eventually be reduced to predominantly monomers. It is noteworthy that, depending on the chemical constituents, crystallization of PC materials can be nucleation dominated or growth dominated. In the former case the prevalent mechanism of crystallization is the stochastic formation of new nuclei and their subsequent growth. For growth-dominated crystallization one observes deterministic growth of neighboring crystallized material into the amorphous region. An important role is also played by any crystal clusters which exist in a melt-quenched amorphous state.

There are several approaches to modeling the processes of crystallization and amorphization during the phase-change processes. One of the most popular and useful is the Johnson-Mehl-Avrami-Kolmogorov (JMAK) model [4-6], which attempts to predict the fraction of crystallized material at every given point in time. This model assumes that the nucleation occurs randomly and uniformly, and the nucleation rate is time independent. The validity of the JMAK model has been recently investigated [7-10], and several arguments have been forwarded as attempts to explain differences between its predictions and observed experimental results. One principal problem is that experimental evidence suggests that many underlying assumptions of the JMAK model are violated: the nucleation takes place mainly on the boundary between the phase-change material and the surrounding substrates $[11,12]$ rather then uniformly and, furthermore, there exist non-negligible incubation times preceding the onset of crystallization [13]. Although JMAK cannot give information about the distribution of cluster sizes, it is a useful model for studying total crystallization.

A more sophisticated alternative to the JMAK model is an approach based on a so-called master equation (also known as the Zeldovich equation) used to describe the crystallization dynamics of PC materials $[14,15]$. Within this framework the dynamics is determined by the temporal evolution of a cluster size distribution function, which is the density of material in clusters of various sizes. Rate equations are used to obtain the frequencies of attachment and detachment of monomers, which represent unit changes in cluster sizes $[14,16,17]$. Several techniques have been employed in order 
to study the master equation in the special cases of small or large cluster sizes together with clusters near the critical nuclei size (see Refs. [18-21] and references therein). Although the steady states can be examined analytically $[18,19]$, in the transient regime (which is particularly relevant for data storage applications), the approximations used in these approaches are invalid. More recently, Shneidman has used a combination of Laplace transform and matched asymptotics to obtain the large-time behavior of nucleation fluxes and cluster size distributions. An interesting review of various thermodynamic aspects of the master equation and their influence on cluster size distributions can be found in Ref. [22].

In this paper we develop a different formulation of the master equation in which we assume a continuous distribution of clusters of sizes $n \geqslant 2$ while retaining a separate scalar for the monomer $n=1$ concentration. The set of continuous and discrete equations are closed by applying appropriate boundary conditions at $n=2$ and $\infty$ and using an integral to incorporate the exhaustion of monomer. An advantage of this is we can readily examine the effect of monomer exhaustion critical in the dynamics of the cluster distribution. Several important assumptions are incorporated within our system. In particular, the heterogeneity of nucleation is accounted for by a "spherical cap model" which allows one to represent the surface interaction of PC material and its substrates by the so-called wetting angle [18]. This angle is related to the specific surface energies of substrate $/ n$-sized cluster and substrate/bulk new phase interfaces. The heat transfer can be modeled directly with a thermal equation whose solution determines the local crystallization regime. This is particularly important when modeling the recrystallization of amorphous marks, since the temperature gradients can be quite significant.

We illustrate the applicability of our model by simulating several isothermal and ramped anneals of $\mathrm{Ge}_{2} \mathrm{Sb}_{2} \mathrm{Te}_{5}$ material (GST). Although this is a multicomponent alloy, the investigations of a number of authors (e.g., Refs. [23-25]) and the speed with which reversible changes can appear in this material suggests that diffusive separation of elements does not play a role in the crystallization process. It therefore is reasonable to consider the material as composed of "monomer" molecules that may or may not be aligned with adjacent monomers to form a crystal structure. The master equation approach in its fully discrete form has been recently used to model some of the experiments on crystallization of GST [26]. However, a drawback of the numerics is that it entails the solution of systems of differential equations with hundreds or thousands of variables. This is computationally very intensive. We show how this disadvantage can be overcome with the continuous formulation of the master equation leading to a problem that can be efficiently handled by modern numerical solvers and demonstrate some examples of anneals that are appropriate to write and erase processes in optical memories.

\section{DERIVATION OF THE MODEL}

As mentioned in the Introduction we characterize the crystallization by a time-dependent cluster size density
$Z(n, \mathbf{x}, t)$ which denotes the probability that at time $t$ the point $\mathbf{x}$ is a part of a size $n$ cluster of crystallized material. We consider a continuum for $n \geqslant 2$ and set

$$
Z_{m}=Z(1, \mathbf{x}, t)
$$

to be the density of monomer. We will use this quantity to measure the proportion of crystallized material and the progress of the crystallization. We define the proportion of crystallized material in some sample (or size large compared to crystal size) to be the ratio of monomers that are in size $n$ clusters $(n \geqslant 2)$ to the total monomers in that sample. The relationship between this definition of crystallinity and material properties such as reflectivity or electrical conductance is not necessarily linear or simple [27].

The temporal dynamics of $Z(n, \mathbf{x}, t)$ (for $n \geqslant 2$ ) and $Z_{m}$ is governed by the master equation which describes the kinetics of a crystallization process. We use a generalization of the Szilard model, i.e., it is assumed that formation of crystal clusters is a result of successive attachments and/or detachments of single molecules (monomers). The detachment frequency is hard to specify accurately as it depends on the cluster properties which are themselves poorly known since the cluster is a new phase of finite size [18]. We use a standard approach to circumvent this difficulty by introducing a quasiequilibrium cluster size distribution function $C(n, T)$ [15], and use this to approximate the detachment frequency from attachment frequency. We retain the $\mathbf{x}$ dependence in the formulation of the master equation approach but note that in this paper we only consider bulk (i.e., space-independent) simulations.

\section{A. The master equation}

The master equation for evolution of the cluster size density $Z(n, \mathbf{x}, t)$ is

$$
\frac{\partial}{\partial t} Z(n, \mathbf{x}, t)=\frac{\partial}{\partial n}\left\{f\left(n, T, Z_{m}(\mathbf{x}, t)\right) C(n, T) \frac{\partial}{\partial n}\left[\frac{Z(n, \mathbf{x}, t)}{C(n, T)}\right]\right\}
$$

for $n \geqslant 2$, which is a time- and space-dependent version of the master equation derived by Zeldovich for isothermal nucleation at constant supersaturation [15]. In Eq. (1) $f\left(n, T, Z_{m}(\mathbf{x}, t)\right)$ is the frequency of monomer attachment to an $n$-sized cluster at temperature $T$ and, naturally, it depends on the concentration of available monomer $Z_{m}(\mathbf{x}, t)$. Further, $C(n, T)$ is the above-mentioned quasiequilibrium cluster size distribution and it is supposed that the spatial dynamics of the cluster size distribution function $Z$ is driven by spatial variations in $f$ and $C$ which are in turn determined by variations in $T$.

The total density of crystalline material can be expressed as

$$
\int_{n=2}^{\infty} n Z(n, \mathbf{x}, t) d n
$$

which must remain finite at all times and, on obvious physical grounds, we note that $Z(n, \mathbf{x}, t) \geqslant 0$. The density of mono- 
mer $Z_{m}$ is determined by the conservation of material

$$
Z_{m}(\mathbf{x}, t)=Z_{0}-\int_{2}^{\infty} n Z(n, \mathbf{x}, t) d n
$$

where $Z_{0}$ is the total density of material, assumed to be constant independent of phase.

The master equation (1) must be augmented by appropriate boundary conditions. For large $n$ we require

$$
\lim _{n \rightarrow \infty} Z(n) \rightarrow 0,
$$

in keeping with the condition of bounded total density of cluster sizes [note that $C(n, T)$ does not satisfy this in undercooled melts]. A suitable boundary condition at $n=2$ can be derived by the following argument. Let

$$
N=\int_{2}^{\infty} Z(n, \mathbf{x}, t) d n
$$

be the total density of crystallites of various sizes (excluding monomers) located at the point $\mathbf{x}$ at time $t$. In view of master equation (1) the time derivative of this quantity is given by

$$
\begin{aligned}
\frac{\partial N}{\partial t} & =\int_{2}^{\infty} \frac{\partial}{\partial t} Z(n, \mathbf{x}, t) d n \\
& =\left.\left[f\left(n, T, Z_{m}\right) C(n, T) \frac{\partial}{\partial n} \frac{Z(n, \mathbf{x}, t)}{C(n, T)}\right]\right|_{n=2} ^{n=\infty} \\
& =-f\left(2, T, Z_{m}\right) C(2, T)\left[\frac{\partial}{\partial n} \frac{Z(n, \mathbf{x}, t)}{C(n, T)}\right]_{n=2} .
\end{aligned}
$$

On the other hand the rate of change in $N$ is determined by the rate of creation or destruction of monomers - such events occur when monomers attach to other monomers or when monomers are detached from dimers (recall the underlying assumption that the size of a cluster can only change by one at each step). Hence

$$
\frac{\partial N}{\partial t}=f\left(1, T, Z_{m}\right) Z_{m}(\mathbf{x}, t)-d\left(2, T, Z_{m}\right) Z(2, x, t) .
$$

The detachment frequency $d$ can be expressed in terms of attachment frequency $f$ via the quasiequilibrium cluster size distribution function $C(n, T)$ as in Ref. [18],

$$
f\left(1, T, Z_{m}\right) C(1, T)=d\left(2, T, Z_{m}\right) C(2, T) .
$$

(This relationship is only formally justifiable in close-toequilibrium situations and, in particular it can lead to an unrealistic dependence of $d$ on $Z_{m}$.) Using Eq. (6) in Eq. (5) and then Eq. (4) gives

$$
\begin{aligned}
& -\left.f\left(2, T, Z_{m}\right) \frac{\partial}{\partial n} Z(n, \mathbf{x}, t)\right|_{n=2} \\
& \quad+\left[f\left(1, T, Z_{m}\right) F_{1}(T)+f\left(2, T, Z_{m}\right) F_{2}(T)\right] Z(2, \mathbf{x}, t) \\
& =f\left(1, T, Z_{m}\right) Z_{m}(\mathbf{x}, t),
\end{aligned}
$$

where

$$
F_{1}(T) \equiv \frac{C(1, T)}{C(2, T)},\left.\quad F_{2}(T) \equiv \frac{\partial}{\partial n} \ln C(n, T)\right|_{n=2} .
$$

This boundary condition, together with Eq. (3), allows one to study the temporal evolution of $Z$ as an initial value problem with a given initial cluster size distribution $Z(n, 0)$.

\section{B. Thermodynamics of cluster formation}

Attachment of monomers is controlled by mass transfer subject to an appropriate activation energy. Let $W(n, T)$ be the work required for the heterogeneous formation of a size $n$ cluster at temperature $T$. Following Ref. [26] this can be written as

$$
W(n, T)=-K_{1}(T) n+K_{2} n^{2 / 3}
$$

where the coefficients can be interpreted in the following way. First, $K_{1}(T)$ is the supersaturation, i.e., the difference (per monomer) in the bulk Gibbs free energy between the old and the new phases while $K_{2}$ (which we assume to be temperature independent) describes the difference in the surface energy between the two phases. The quantity $K_{1}(T)$ has been approximated by Singh and Holz [28] as

$$
K_{1}(T)=v_{0} \Delta H_{f}\left(\frac{T_{m}-T}{T_{m}}\right)\left[\frac{7 T}{T_{m}+6 T}\right],
$$

where $v_{0}$ is the volume of a monomer, $T$ is current temperature, $T_{m}$ is the melting temperature of the PC material, and $\Delta H_{f}$ is the enthalpy of fusion at the melting point.

The coefficient $K_{2}$ has the form

$$
K_{2}=\left[\psi\left(\theta_{w}\right)\right]^{2 / 3} a \sigma
$$

with $a \equiv\left(36 \pi v_{0}^{2}\right)^{1 / 3}$ and $\sigma$ the specific surface energy of the planar new phase/old phase interface. To allow for heterogeneous nucleation, we assume that nucleating crystals form caps on the surface of the substrate with some wetting angle $\theta_{w}$. The geometrical factor $\psi\left(\theta_{w}\right)$ takes a value somewhere between $\psi(0)=0$ and $\psi(\pi)=1$ and, according to Ref. [18], assumes the particular form

$$
\psi\left(\theta_{w}\right)=\frac{1}{4}\left(2+\cos \theta_{w}\right)\left(1-\cos \theta_{w}\right)^{2} .
$$

Choosing appropriate values for $\theta_{w}$ thus enables heterogeneous or homogeneous nucleation to be modeled.

Observe that $K_{1}$ changes sign at $T=T_{m}$ : below the melting point $T<T_{m}$ we have $K_{1}>0$ and there is a critical cluster size given by

$$
n^{*}=\frac{8}{27}\left(\frac{K_{2}}{K_{1}}\right)^{3}
$$

i.e., $n^{*}$ such that $d W\left(n^{*}, T\right) / d n=0$.

\section{Approximation of $f$ and $C$ in the master equation}

For our particular intended applications the latent heat released during a phase transition can be neglected since the amount of heat conducted through the substrate is signifi- 
cantly greater. Since we consider nucleation of crystals in the melt, the main mechanism of mass transport that governs the attachment of monomers to the existing clusters, is that of interface control [18]. In this process the monomers to be attached are supposed to be in the close proximity of the condensed-phase cluster and can join the cluster by making a random jump over a distance comparable with their diameter [29]. The attachment frequencies for the three-dimensional heterogeneous nucleation of caps can be approximated as

$$
f\left(n, T, Z_{m}\right)=\hat{f} Z_{m} n^{2 / 3} \exp \{-[W(n+1, T)-W(n, T)] / k T\},
$$

where $k$ is the Boltzmann constant and $\hat{f}$ is given by

$$
\hat{f}=\frac{c v_{0}^{2 / 3}\left(1-\cos \theta_{w}\right)}{2 d_{0} \psi^{2 / 3}\left(\theta_{w}\right)} D .
$$

Within this formula the geometrical function $\psi$ is as in Eq. $(10), d_{0}$ and $v_{0}$ denote the diameter and volume of the monomer, respectively, and $c$ is a "shape factor" for heterogeneous nucleation; it is $(36 \pi)^{1 / 3}$ for the case of caps. Finally $D$ is a diffusion coefficient which may also be space and time dependent through its dependence on the temperature; see for example Ref. [26].

The quasiequilibrium cluster size distribution $C(n, T)$ depends on the temperature [18] and is given simply by

$$
C(n, T)=\exp \{-W(n, T) / k T\}
$$

up to a multiplicative constant that is independent of $n$. Substituting the form (8) for $W(n, T)$ shows that

$$
C(n, T)=\exp \left\{\left[K_{1}(T) n-K_{2} n^{2 / 3}\right] / k T\right\} .
$$

The specific forms of the attachment frequency (11) and quasiequilibrium cluster size distribution (13) when substituted into the master equation (1) give rise to a FokkerPlanck equation of the type

$$
\frac{\partial Z}{\partial t}=\frac{\partial}{\partial n}\left(v\left(n, T, Z_{m}\right) Z(n, \mathbf{x}, t)+f\left(n, T, Z_{m}\right) \frac{\partial Z(n, \mathbf{x}, t)}{\partial n}\right),
$$

where

$$
v\left(n, T, Z_{m}\right)=-f\left(n, T, Z_{m}\right) \frac{\partial}{\partial n}[\ln C(n, T)]
$$

as in [Ref. [18], Eq. (9.29)].

\section{Evolution of temperature field}

The temporal dynamics of the temperature is governed by the heat equation

$$
\rho C \frac{\partial T}{\partial t}-\kappa \Delta T=g,
$$

where $\rho$ is a material density, $C$ is a specific heat, $\kappa$ is a thermal conductivity, and $g$ is a bulk heat source or sink. We note that these material properties may change in quite com- plicated ways as the proportion of the different phases change.

Equations (14) and (15) together with the boundary conditions (2), (3), and (7) form a closed system of equations that determine the dynamics of the spatial density $Z(n, t)$ of clusters of various sizes and a (possibly) time-dependent temperature field $T$. Although for fixed $Z_{m}$ this system is linear in $Z$, the dependencies on $n$ are too involved for analytical solution, though we discuss some particular limiting cases separately.

\section{ISOTHERMAL LIMIT OF LARGE CLUSTER SIZES}

We begin our analysis of the system by considering the case when $T(\mathbf{x}, t)=$ const and restricting to large values of $n$. In this approximation two particular cases are of interest. The first corresponds to the situation when the latter term in the expression (8) dominates. Physically, this situation describes the case when the temperatures are high, and consequently, the size of the critical nuclei is large. We restrict ourselves to the initial stage of nucleation when it is reasonable to take $Z_{m}(\mathbf{x}, t) \approx Z_{0}=$ const. In this regime, for large cluster sizes 2 $\ll n \ll n^{*}$, the master equation reduces to a linear partial differential equation (PDE) with coefficients depending on $n$ : in particular

$$
\frac{\partial Z}{\partial t}=\frac{\partial}{\partial n}\left\{\exp \left(-\frac{2}{3} \frac{p_{1}}{p_{2}} n^{-1 / 3}\right)\left[p_{1} n^{1 / 3} Z+p_{2} n^{2 / 3} \frac{\partial Z}{\partial n}\right]\right\},
$$

where

$$
p_{1} \equiv \frac{2}{3} \frac{K_{2}}{k T} \hat{f} Z_{m} \exp \left(\frac{K_{1}}{k T}\right) \quad \text { and } \quad p_{2} \equiv \hat{f} Z_{m} \exp \left(\frac{K_{1}}{k T}\right),
$$

are independent of $n$ and $t$. Changing variables to $n=a^{3 / 2}$ and $V(r, t)=n^{1 / 3} Z$, one obtains in the limit of large cluster sizes $2 \ll n \ll n^{*}$ the equation

$$
\frac{\partial V}{\partial t}=\frac{2}{3} p_{1} \frac{\partial V}{\partial a}-\frac{2}{9} p_{2} \frac{\partial}{\partial a}\left(\frac{V}{a}\right)+\frac{4}{9} p_{2} \frac{\partial^{2} V}{\partial a^{2}} .
$$

Separation of variables in Eq. (17) gives the eigenmodes of the solution of this equation as

$V_{\lambda}^{1}(a, t)=a^{1 / 4} \exp \left[\lambda t-3 p_{1} a /\left(4 p_{2}\right)\right] M_{\kappa, \mu}\left(3 a \sqrt{p_{1}^{2}+4 \lambda p_{2}} /\left(2 p_{2}\right)\right)$

$V_{\lambda}^{2}(a, t)=a^{1 / 4} \exp \left[\lambda t-3 p_{1} a /\left(4 p_{2}\right)\right] W_{\kappa, \mu}\left(3 a \sqrt{p_{1}^{2}+4 \lambda p_{2}} /\left(2 p_{2}\right)\right)$

where $W_{\kappa, \mu}$ and $M_{\kappa, \mu}$ are Whittaker functions [30] with

$$
\kappa=\frac{1}{4} \frac{p_{1}}{\sqrt{p_{1}^{2}+4 \lambda p_{2}}} \text { and } \mu=\frac{1}{4} .
$$

With an appropriate choice of boundary conditions it should be possible to form a basis of $L^{2}$ using Eq. (18) for a discrete sequence of eigenfunctions with $\lambda_{n} \leqslant 0$. However, we do not do this here other than to note that the eigenmodes $V_{\lambda}^{k}$ with $\lambda \leqslant 0$ decay exponentially in $a$ for fixed $t$. This agrees with the phenomenologically derived exponential scaling at onset as proposed by Mulheran in the study of continuous nucleation [31]. 
TABLE I. Temperature-independent parameters used for the numerical simulations.

\begin{tabular}{lcc}
\hline \hline \multicolumn{1}{c}{ Parameter } & Value & Reference \\
\hline Volume of monomer $v_{0}$ & $2.9 \times 10^{-28} \mathrm{~m}^{-3}$ & {$[26]$} \\
Specific surface energy $\sigma$ & $0.1 \mathrm{~J} / \mathrm{m}^{2}$ & {$[26,32]$} \\
Enthalpy of fusion $\Delta H_{f}$ & $6.18 \times 10^{8} \mathrm{~J} / \mathrm{m}^{3}$ & {$[26]$} \\
Melting temperature $T_{m}$ & $889 \mathrm{~K}$ & {$[33]$} \\
\hline \hline
\end{tabular}

Another limit of interest is the one corresponding to the regime $2 \ll n^{*} \ll n$, where the bulk contribution in Ref. [8] dominates and, under approximations as above (i.e., at the initial stages of crystallization), Eq. (14) reduces to

$$
\frac{\partial Z}{\partial t}=\frac{\partial}{\partial n}\left[-q_{1} n^{2 / 3} Z+q_{2} n^{2 / 3} \frac{\partial Z}{\partial n}\right],
$$

with $q_{1}$ and $q_{2}$ given by

$$
q_{1}=\frac{\hat{f} Z_{m} K_{1}}{k T} \exp \left[\frac{K_{1}}{k T}\right] \text { and } q_{2}=\hat{f} Z_{m} \exp \left[\frac{K_{1}}{k T}\right] .
$$

A steady state of this equation is easily seen to be

$$
Z(n) \sim \exp \left[\frac{q_{1}}{q_{2}} n\right],
$$

and it coincides with the large- $n$ asymptotic form of the equilibrium cluster size distribution $C(n)$.

Changing variables to cluster radius $r$, where $n=r^{3}$ and $V(r, t)=n^{2 / 3} Z(n, t)$, gives

$$
\frac{\partial V}{\partial t}=-\frac{p_{1}}{3} \frac{\partial V}{\partial r}+\frac{p_{2}}{9} \frac{\partial^{2}}{\partial r^{2}}\left[\frac{V}{r^{2}}\right] .
$$

The general steady solution of Eq. (20) can be written in terms of Whittaker functions as

$$
\begin{aligned}
V(r)= & e^{q_{1} r^{3} / q_{2}}\left\{C_{1} r^{2}+\frac{C_{2}}{\sqrt{r}}\left[3 r^{2} M_{1 / 6,2 / 3}\left(\frac{q_{1} r^{3}}{q_{2}}\right)\right.\right. \\
& \left.\left.+4 q_{2} W_{1 / 6,2 / 3}\left(\frac{q_{1} r^{3}}{q_{2}}\right)\right]\right\} .
\end{aligned}
$$

In principle the constants $C_{1,2}$ can be deduced by imposition of appropriate boundary conditions. Unlike the previous case however, it appears that a complete set of time-dependent eigenfunctions of Eq. (20) cannot be obtained analytically and numerical simulations have to be resorted to instead.

\section{NUMERICAL SIMULATIONS}

Various physical characteristics of GST material are incorporated in our model based on known properties of the GST alloy $\mathrm{Ge}_{2} \mathrm{Sb}_{2} \mathrm{Te}_{5}$ commonly used in phase change optical and electrical memories. These parameter values are listed in Table I. As discussed in Ref. [26], several of these values have to be determined indirectly from results of experiments on composite materials similar to GST, since for
GST itself they have not yet been measured. The diffusion coefficient $D$ was obtained from the Stokes-Einstein relation

$$
D=\frac{k T}{3 \pi \lambda \eta}
$$

where $\lambda$, the jump distance, can be well approximated by the GST nearest-neighbor distance $(2.99 \AA)$. The viscosity $\eta$ has the Arrhenius type of temperature dependence,

$$
\eta=K_{\eta} \exp \left[\frac{E_{a}}{k T}\right]
$$

where the activation energy for GST has been estimated as $E_{a} \approx 2 \pm 0.1 \mathrm{eV}[13,26]$ and the prefactor $K_{\eta}=5.82$ $\times 10^{-12} \mathrm{~m}^{2} / \mathrm{s}$ was found by fitting to obtain the correct incubation time at $130{ }^{\circ} \mathrm{C}$. However, observe that even a small uncertainty in $E_{a}$ translates into a very large uncertainty in $K_{\eta}$. Quite significant variations in the values of wetting angle have been reported for different substrates; we take $\theta_{w}=94^{\circ}$ previously used in simulations of crystallization on silicon oxide substrate [26].

The system (1)-(3) and (7) was solved using the FORTRAN NAG routine D03PSF. This routine uses a backward difference approximation for convection-diffusion equations with adaptive remeshing: this is particularly important for our problem due to exponential behavior of the cluster size distribution density at dimers and the boundary layer that forms at maximum cluster size. We performed our simulations on a finite interval $[2, L]$ with a variety of values of $L$ and space and time discretizations. The results presented here mostly relate to the length $L=40$ divided up into 100-500 subintervals. We used the adaptive time step recommended by the routine for absolute and relative tolerances $10^{-10}$. This gave a very wide range of time steps, ranging from less than $10^{-10} \mathrm{~s}$ to $1 \mathrm{~s}$ depending on current system state and parameters. The scale of time evolution for the problem depends very sensitively on the temperature and the state of the distribution; nonetheless we could obtain reliable results over a range of temperatures up to over melting temperature using just a few minutes on a personal computer. Before each time step $Z_{m}$ was set according to Eq. (2). The initial condition imposed on the system was that of an undercooled melt in which all matter is concentrated in monomers; this is a reasonable model for as-deposited PC material on an optical disk.

We begin our account of the simulations with some isothermal treatments. An important quantitative characteristic of phase-change processes in GST is the crystallized fraction, which signifies how much of the total volume of undercooled melt has been already crystallized. In experiments the amount of crystallized material is usually determined by measuring the change in optical properties of the GST, for instance its reflectance. For isothermal anneals these measurements can be performed in real time for low temperatures [13]. The results of our simulations are shown in Fig. 1 for the values of the annealing temperature $T=119,131$, and $140{ }^{\circ} \mathrm{C}$. These results are in good agreement with the experimental data [13] and the simulations with a discrete version of master equation [26]. The incubation time is estimated to be the time taken until an arbitrary proportion (50\%) of the 


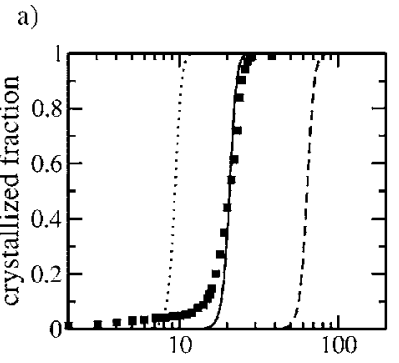

time (min)

b)

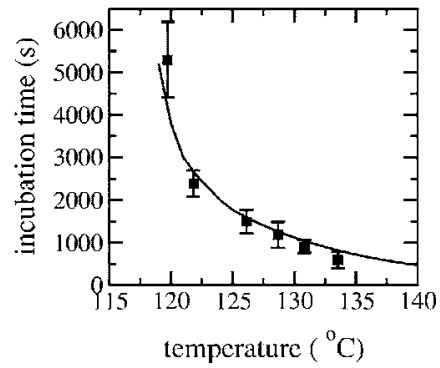

FIG. 1. (a) Crystallization behavior of GST during isothermal anneals. The solid denote simulation results and symbols are experimental data from Ref. [13] for $T=131{ }^{\circ} \mathrm{C}$; the dashed line shows $T=119^{\circ} \mathrm{C}$ and the dotted line $T=140{ }^{\circ} \mathrm{C}$. (b) Dependence of incubation time on the annealing temperature. The line shows simulation result. Symbols are experimental data taken from Ref. [13].

monomer has been crystallized. This gives an estimate of the length of the period before which the dynamics becomes governed by the steady-state nucleation and growth. For the temperatures investigated, the incubation times are very large, and therefore for data storage purposes, the process of crystallization takes place in the regime of transient nucleation [19]. This suggests the importance of using an explicitly time-dependent master equation rather than the JMAK approach with assumed stationary nucleation and growth rates.

In Fig. 2(a) we show several initial iterations of the master equation for $T=140{ }^{\circ} \mathrm{C}$. This figure suggests that the monomers start forming clusters at an early stage, which subsequently grow into the clusters of larger sizes. Qualitatively, this is in good agreement with Eq. (18). Later, the original pool of monomers is being exhausted, and a redistribution of cluster sizes takes place with larger clusters gaining further monomers and smaller clusters losing them. Eventually the system settles on a distribution shown in Fig. 2(b). This curve is in good qualitative agreement with the phenomenological expression for cluster size distribution in the case of nucleation which occurs as site saturation: $Z(n)$ $=n^{\alpha-1} \alpha^{\alpha} e^{-\alpha n} / \Gamma(\alpha)$ [34]. Site saturation means that all nuclei are simultaneously present and just grow, as compared to the mechanism of continuous nucleation when new nuclei appear at a certain rate.

a)
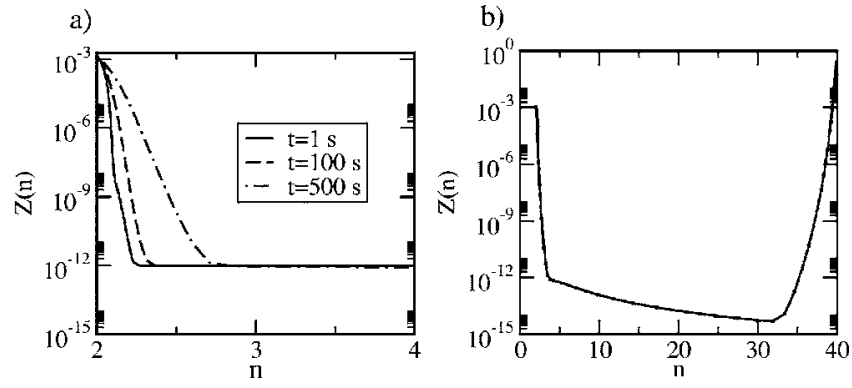

FIG. 2. Numerical solutions of the master equation (1). (a) Initial iterations for $T=140{ }^{\circ} \mathrm{C}$. (b) Final cluster size distribution profile for $T=140{ }^{\circ} \mathrm{C}$-the final profile is determined by the exhaustion of monomer.

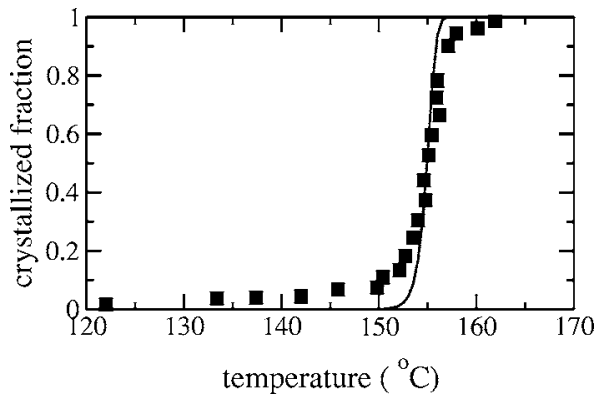

FIG. 3. Crystallized fraction of the GST material as a function of temperature during a ramped anneal with a heating rate $3{ }^{\circ} \mathrm{C} / \mathrm{min}$ starting at $120^{\circ} \mathrm{C}$. The line shows the result of simulation using our model, while the squares are experimental data from Ref. [35].

The main problem for data storage media concerns the dynamics of crystallization behavior during nonisothermal anneals. Ramped temperature anneals are often used to gain insight into the dynamic behavior of phase change materials. We briefly describe our simulations of the effect of a constant temperature ramp on fraction of crystallized material. First we consider the case when the temperature gradually increases from an initial temperature $T=120^{\circ} \mathrm{C}$ with a constant ramp rate $3{ }^{\circ} \mathrm{C} / \mathrm{min}$; results are presented in Fig. 3, and these show a crystallization curve which is in good agreement with experimental results. We have also performed simulations of the cooling of the GST material starting from the melting temperature. If the cooling rate is sufficiently slow then the material fully crystallizes; if it is sufficiently fast then the material effectively reamorphizes, while intermediate cooling rates can result in an arbitrary proportion of residual crystalline material. This should enable one to undertake more careful numerical modeling of the "priming" of GST [23].

As a final example we consider a nonisothermal process starting at an amorphous initial state with $T=100{ }^{\circ} \mathrm{C}$ at $t$ $=0$. At $t=1 \mathrm{~s}$ we change to $T=240{ }^{\circ} \mathrm{C}$ and return to $T$ $=100{ }^{\circ} \mathrm{C}$ at time $t=1.1 \mathrm{~s}$. At time $t=1.2 \mathrm{~s}$ we raise the temperature to $T=500{ }^{\circ} \mathrm{C}$ for a time $\tau$ before returning to $100{ }^{\circ} \mathrm{C}$. Figure 4 shows (a) the evolution of crystallization fraction and (b) the final distribution of cluster sizes at $t$ $=1.3 \mathrm{~s}$ for this anneal with $\tau=0.1$ and $0.2 \mathrm{~ms}$. One can clearly see that the final crystalline fractions are very different, and this is reflected also in the distribution of cluster sizes (b) at the end of the process. For the larger $\tau$ the material is effectively reamorphized.

\section{DISCUSSION}

The dynamics of crystallization behavior in phase-change materials has been considered using a master-equation approach. A major advantage of the master-equation technique is that, unlike simpler methods, it gives fast prediction of cluster size distributions from the thermodynamics of the crystallizing material. In particular, it does not require additional assumptions about the phase change processes implicit in the justification of the JMAK model. These assumptions 

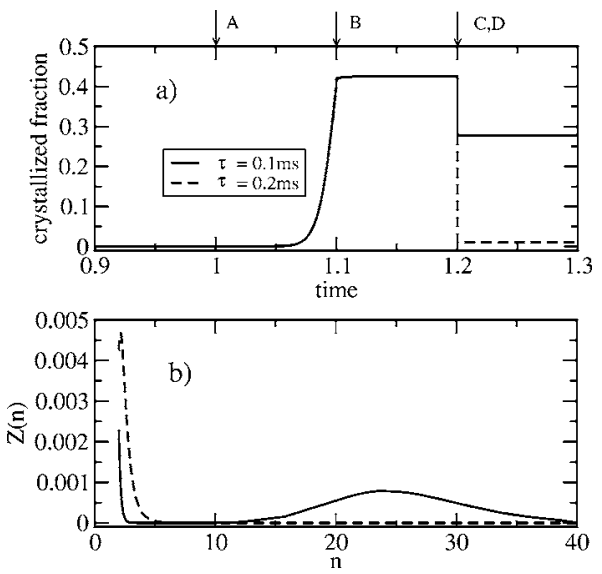

FIG. 4. (a) Crystallized fraction of the GST material and (b) distribution of crystal sizes for two different anneals; during $A$ to $B$ the material is subjected to $240{ }^{\circ} \mathrm{C}$ for $0.1 \mathrm{~s}$ while between $C$ and $D$ it is subjected to $500{ }^{\circ} \mathrm{C}$ for a very short time $\tau$ (see text).

include among others homogeneity and the randomness of crystallization as well as the stationarity of nucleation.

There are of course other alternatives to the JMAK model that predict the cluster size distribution and we describe briefly two of these. Phase-field methods (see, e.g., Ref. [36] and references therein) are effective in the modeling of the crystal growth in a melt, but are computationally very intensive. Another option is direct molecular simulation, but this is even more demanding, especially if one wishes to attempt simulations using sufficiently many molecules to derive a realistic distribution of crystal sizes. In addition, some insight into the dynamics of crystallization can be gained from the analytical solution of the master equation in particular cases.
We have performed numerical simulations of isothermal and nonisothermal treatments of GST. The results of these computations are in good agreement with experimental observations. Our subsequent research will concentrate on further development of the master-equation approach and its extension to the study of crystallization dynamics in the presence of nonhomogeneities, i.e., when the spatial effects start to be important. This will require the simultaneous solution of both the master equation and a coupled heat equation for the temperature field. Still, since the master equation does not include explicit spatial derivatives, we will obtain an approximation of the crystallite size distribution much more rapidly than if phase-field or direct molecular dynamics simulations were used.

In our numerical simulations the initial condition for cluster distribution function has been taken to be an as-deposited pool of monomers with no larger clusters present. From both experimental and practical points of view it is interesting to investigate the influence of preexisting clusters on the final cluster size distribution [3]. This will give insight into the mechanisms occurring in phase-change processes that are the result of several heating-cooling cycles. We believe this could shed light on the most efficient way to achieve a desired distribution of cluster sizes and, correspondingly, help optimize the write-erase and initialization processes in data storage media. It could also prove useful in understanding the behavior of the so-called cognitive computer [27,37], at whose heart is a multi-input phase-change cell that relies on accumulation of crystallized material to provide a thresholding function analogous to that of a neuron.

\section{ACKNOWLEDGMENTS}

This work was supported by EPSRC Grant No. GR/ S31662/01.
[1] M. Libera and M. Chen, MRS Bull. 15, 40 (1990).

[2] J. Tominaga, T. Kikukawa, M. Takahashi, T. Kato, and T. Aoi, Jpn. J. Appl. Phys., Part 1 36, 3598 (1997).

[3] P. K. Khulbe, E. M. Wright, and M. Mansuripur, J. Appl. Phys. 88, 3926 (2000).

[4] M. Avrami, J. Chem. Phys. 7, 1103 (1939); M. Avrami, ibid. 8, 212 (1940);M. Avrami, ibid. 9, 177 (1941).

[5] W. A. Johnson, and R. F. Mehl, Trans. AIME 135, 416 (1939).

[6] A. N. Kolmogorov, Bull. Acad. Sci. USSR Mat. Ser. 1, 355 (1937).

[7] M. Castro, F. Domínguez-Adame, A. Sánchez, and T. Rodríguez, Appl. Phys. Lett. 75, 2205 (1999).

[8] V. Erukhimovitch and J. Baram, Phys. Rev. B 50, 5854 (1994); V. Erukhimovitch, J. Baram, ibid. 51, 6221 (1995).

[9] M. Fanfoni and M. Tomellini, Phys. Rev. B 54, 9828 (1996).

[10] C. W. Van Siclen, Phys. Rev. B 54, 11845 (1996).

[11] N. Ohshima, J. Appl. Phys. 79, 8357 (1996).

[12] G. Ruitenberg, A. K. Petford-Long, and R. C. Doole, J. Appl. Phys. 92, 3116 (2003).

[13] V. Weidenhof, I. Friedrich, S. Ziegler, and M. Wuttig, J. Appl. Phys. 89, 3168 (2001).
[14] D. Turnbull, and J. C. Fisher, J. Chem. Phys. 17, 71 (1949).

[15] Ya. B. Zeldovich, Zh. Eksp. Teor. Fiz. 12, 525 (1942) [Acta Physicochim. URSS 18, 1 (1943)].

[16] R. Becker and W. Döring, Ann. Phys. 24, 719 (1935).

[17] M. Volmer and A. Weber, Z. Phys. Chem., Stoechiom. Verwandtschaftsl. 119, 277 (1926).

[18] D. Kashchiev, Nucleation (Butterworth-Heinemann, Oxford, 2000).

[19] K. F. Kelton, A. L. Greer, and C. V. Thompson, J. Chem. Phys. 79, 6261 (1983).

[20] V. A. Shneidman, J. Chem. Phys. 115, 8141 (2001).

[21] V. A. Shneidman, J. Chem. Phys. 119, 12487 (2003).

[22] M. Hütter, Multiscale Model. Simul. 1, 371 (2003).

[23] J. H. Coombs, A. P. J. M. Jongenelis, W. van Es-Spiekman, and B. A. J. Jacobs, J. Appl. Phys. 78, 4906 (1995).

[24] J. H. Coombs, A. P. J. M. Jongenelis, W. van Es-Spiekman, and B. A. J. Jacobs, J. Appl. Phys. 78, 4918 (1995).

[25] A. V. Kolobov, P. Fons, A. Frenkel, A. Andudinov, J. Tominaga, and T. Uruga, Nat. Mater. 3, 703 (2004).

[26] S. Senkader and C. D. Wright, J. Appl. Phys. 95, 504 (2004).

[27] D.-H. Kim, F. Merget, M. Laurenzis, P. H. Bolivar, and H. 
Kurz, J. Appl. Phys. 97, 083538 (2005).

[28] H. B. Singh and A. Holz, Solid State Commun. 45, 985 (1983).

[29] J. Frenkel, Kinetic Theory of Liquids (Oxford University Press, Oxford, 1949).

[30] Handbook of Mathematical Functions, edited by M. Abramowitz and I. A. Stegun (Dover, New York, 1972).

[31] P. A. Mulheran, Acta Metall. Mater. 42, 3589 (1994).

[32] B. Hyot, V. Gehanno, B. Rolland, A. Fargeix, C. Vannufel, F. Charlet, B. Béchevet, J. M. Bruneau, and P. J. Desre, J. Magn.
Soc. Jpn. 25, 414 (2001).

[33] N. Yamada, E. Ohno, K. Nishiuchi, N. Akahira, and M. Takao, J. Appl. Phys. 69, 2849 (1991).

[34] D. Weaire, J. P. Kermode, and J. Wejchert, Philos. Mag. B 53, L101 (1986).

[35] T. P. Leervad Pedersen, J. Kalb, W. K. Njoroge, D. Wamwangi, M. Wuttig, and F. Spaepen, J. Appl. Phys. 79, 3597 (2001).

[36] A. Karma and W.-J. Rappel, Phys. Rev. E 57, 4323 (1998).

[37] S. Ovshinsky, in Proceedings of EPCOS 2004 Symposium (Balzers, Lichtenstein, 2004). 